\title{
DETERMINACIÓN DE LAS PROPIEDADES MECÁNICAS DE LA CAÑA GUADUA ANGUSTIFOLIA DEL ECUADOR SOMETIDA A ESFUERZOS FLECTORES Y CORTANTES
}

\author{
DETERMINATION OF THE MECHANICAL PROPERTIES OF GUADUA \\ ANGUSTIFOLIA CANE OF ECUADOR SUBJECTED TO BENDING AND SHEAR \\ STRESSES
}

\author{
ANTONIO LA TEGOLA', LUIS OCTAVIO YÉPEZ ROCA², WALTER VICENTE MERA ORTIZ³, PEDRO LUIS CÓRDOVA ALCÍVAR ${ }^{4}$

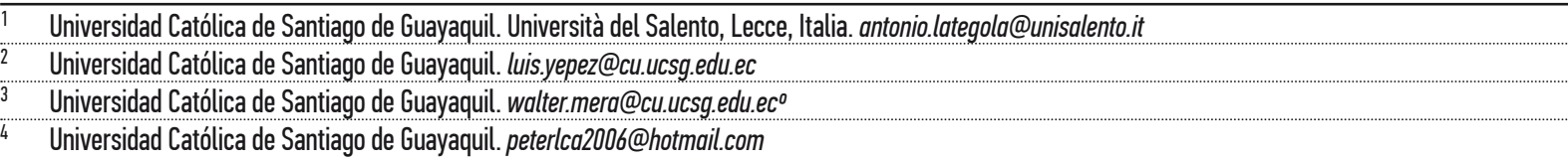

\begin{abstract}
El objetivo es la determinación de las propiedades mecánicas de la caña guadua Angustifolia Kunth de Ecuador cuando está sometida a flexión y esfuerzos cortantes. A través de la metodología experimental se pretendió delimitar los valores de la resistencia al cortante, a la flexión y su módulo de elasticidad. Se realizaron ensayos estructurales a muestras de caña Guadua obtenidas de plantaciones de Valencia, provincia de Los Ríos, Ecuador, para poder dar un soporte ingenieril y científico a este trabajo. Las muestras fueron sometidas a ensayos de corte y flexión. La metodología cuantitativa dio base al trabajo empírico de la investigación, basado en tres variables independientes y ajustadas a través de la revisión bibliográfica. La metodología de diseño se basó en la mecánica estructural, y la norma ISO 22157. El conjunto de las variables será explicado a través del estudio de la información estadística. Los resultados de los ensayos permitieron determinar los valores característicos de las variables de estudio, con los cuales se puede diseñar y construir estructuras de caña Guadua seguras. Con estos y otros resultados producto del trabajo de investigación, los autores esperan aportar a la creación de la nueva norma ecuatoriana de construcción y diseño estructural de bambú.
\end{abstract}

PALABRAS CLAVE: propiedades mecánicas, caña guadua Angustifolia Kunth, propiedades geométricas.
ABSTRACT

The main objective of this research is to determine the mechanical properties of the Bamboo Guadua Angustifolia Kunth of Ecuador, and through the experimental methodology determine the values of the resistance to shear, bending and its modulus of elasticity. Structural tests were made to Guadua samples obtained from plantations in Valencia, province of Los Rios, Ecuador, to provide an engineering and scientific support to these work trials. The samples were subjected to shear and bending. The quantitative methodology given to empirical research work consists of three independent variables and adjusted through the literature review. The design methodology is based on structural mechanics, and the International Standard ISO 22157. The set of variables will be explained through the study of statistical information. The test results allowed determining the characteristic values of the study variables, with which you can design and build safe structures of Guadua cane. According to the authors, these and other results produced by the research can contribute to the creation of the new Ecuadorian standard construction and structural design of bamboo.

KEYWORDS: mechanical properties, Bamboo Guadua Angustifolia Kunth, geometric properties. 
INTRODUCCIÓN

Conscientes del uso estructural de la caña Guadua en los últimos años se han llevado a cabo estudios en diferentes regiones y lugares donde esta es cultivada para determinar sus propiedades y comportamiento mecánico para su correspondiente aplicación en sistemas estructurales.

Bajo la premisa de tecnificación del análisis y diseño de construcciones en Guadua, los autores del presente estudio han considerado pertinente llevar a cabo esta investigación para posteriormente comentar resultados y sugerir recomendaciones de análisis y diseño.

El problema que se plantea resolver es el uso del bambú a través del conocimiento de sus propiedades mecánicas y estructurales, para la elaboración de un procedimiento que permita el uso de este material en el diseño, el cálculo estructural y en la construcción de viviendas.

Es necesario investigar las propiedades mecánicas y estructurales del bambú para la elaboración de una norma de construcción actualmente inexistente en el país, que permita el uso con seguridad estructural del bambú en la construcción de edificaciones. Son necesarias la planificación y ejecución de pruebas de laboratorio en especímenes de bambú para determinar sus propiedades mecánicas y estructurales.

La norma de construcción propuesta del bambú para el Ecuador tiene relación con otras normas de construcción, tales como las normas del hormigón armado, del acero y de la madera en general. La elaboración y creación de una norma de construcción que será de beneficio para los usuarios de la Norma Ecuatoriana de Construcción (NEC).

Las variables a medir son las propiedades del bambú (caña Guadua) a los siguientes esfuerzos: propiedad del bambú a flexión (esfuerzo momento flector); propiedad del bambú a cortante (esfuerzo de corte); módulo elástico del bambú; clasificación de la variación de dimensiones de los especímenes del bambú: ancho, espesor, nudos, etc.

Los valores característicos de la resistencia se calcularon como percentiles de resistencia menor que 5\%, fractiles. Teniendo en cuenta el número de muestras, se hizo referencia a la teoría de pequeñas muestras mediante la estimación de la media y de la varianza. Además se adoptó un intervalo de confianza igual al $75 \%$ con el fin de tener en cuenta la naturaleza del material.

METODOLOGÍA

Caña Guadua Angustifolia Kunth de Ecuador fue usada en los ensayos. Esta caña fue traída desde plantaciones cercanas al cantón Valencia en la provincia de Los Ríos. Otros detalles sobre el origen de estas cañas son desconocidos y ya estaban cortadas en longitudes de aproximadamente seis metros. Habían sido tratadas en soluciones químicas con bórax y ácido bórico y sometidas a procedimientos de secado para mejorar su durabilidad. Estas cañas estaban almacenadas en bodegas de almacenamiento en los galpones de la Planta de Ecomateriales de la Universidad Católica de Santiago de Guayaquil (UCSC), en Durán.

De una población de alrededor de 170 cañas Guadua, aparentemente tallos sanos sin ningún tipo de ataque por insectos o de hongos, huecos o imperfecciones se seleccionaron arbitrariamente más de diez cañas para hacer las muestras para los ensayos.

Para las pruebas de flexión se elaboraron diez muestras y para las pruebas de corte quince muestras. Las pruebas de los ensayos se realizaron en el laboratorio de estructuras de la Facultad de Ingeniería de la UCSG, en el edificio del Centro de Investigaciones en Ingeniería Estructural Sismo-Resistente (CEINVES). Se realizaron algunas adaptaciones a los equipos existentes, debido a que era la primera vez que se hacían este tipo de pruebas.

\section{PREPARACIÓN DE EQUIPOS DE ENSAYOS DE CORTE}

Se elaboraron quince muestras de caña Guadua para estos ensayos, la altura de cada muestra era igual a su medida de diámetro externo, es decir una relación 1:1. De las quince muestras, diez contenían un nudo y cinco sin nudo. El ensayo consistió en aplicar fuerzas de corte a los extremos de las muestras a través de dos placas huecas que formen los planos de falla, tal como se puede apreciar en la figura 1.

En la máquina de compresión se aplicaron fuerzas de compresión a las placas, las cuales transmiten fuerzas de corte a la muestra de caña Guadua paralelas a la fibra. Las figuras 2 y 3 ilustran la ejecución de un ensayo de resistencia al corte. 


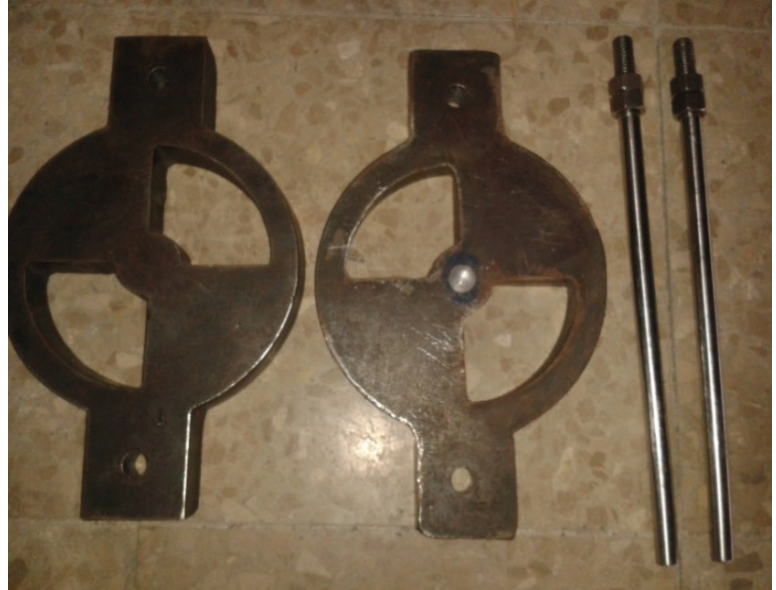

Figura 1. Placas huecas en cuadrantes opuestos para formar los planos de falla en el ensayo de corte.

Fuente: Córdova, P. (2014). Obtención de las propiedades mecánicas y estructurales de la caña Guadua Angustifolia Kunth del Ecuador.

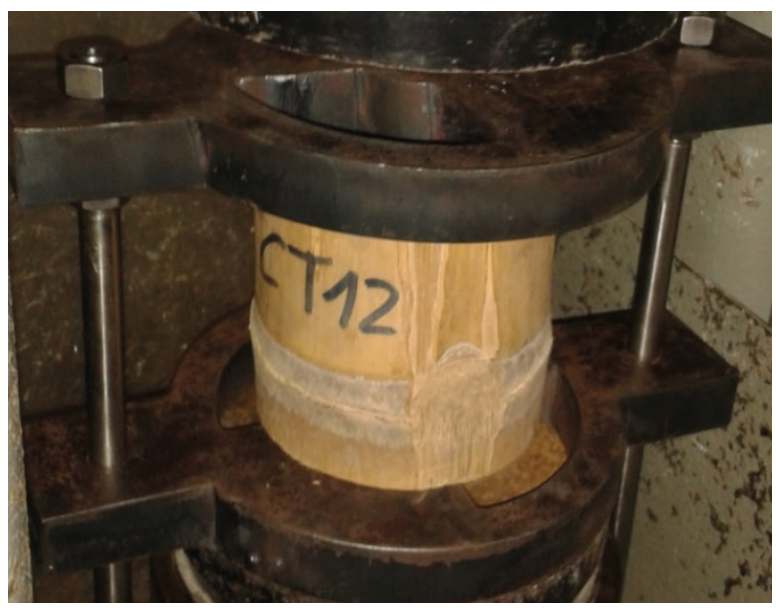

Figura 2. Ensayo de corte en la máquina de compresión simple. Fuente: Córdova, P. (2014). Obtención de las propiedades mecánicas y estructurales de la caña Guadua Angustifolia Kunth del Ecuador.
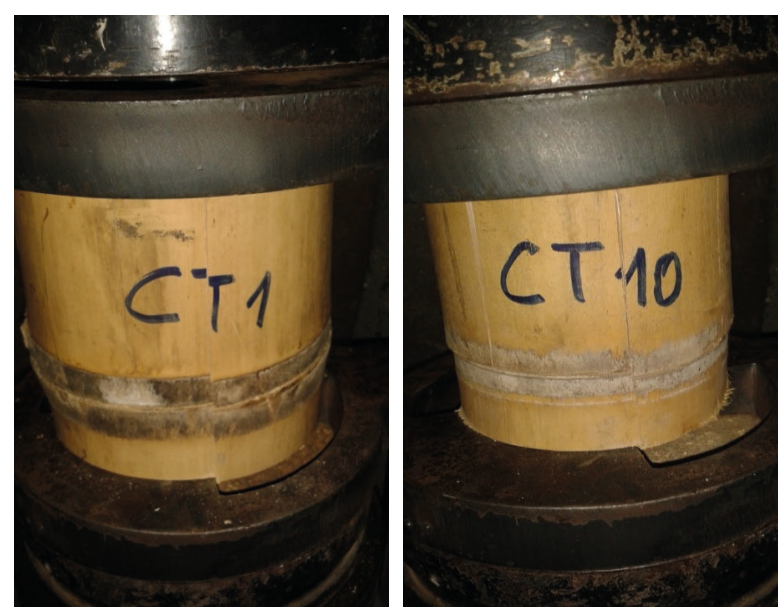

Figura 3. Mecanismo de falla del ensayo de corte al llegar a la resistencia última al esfuerzo cortante.

Fuente: Córdova, P. (2014). Obtención de las propiedades mecánicas y estructurales de la caña Guadua Angustifolia Kunth del Ecuador.

\section{PREPARACIÓN DE EQUIPOS DE ENSAYOS DE FLEXIÓN} Se elaboraron diez muestras de caña Guadua para estos ensayos, la longitud de cada muestra era mayor treinta veces a la medida de su diámetro externo, es decir una relación 30:1. Se requiere estas dimensiones $(\mathrm{L} \geq 30 \mathrm{D})$ para la muestra de forma que permita ensayar a la caña y que se obtenga flexión pura sin esfuerzos ocasionados por fuerzas cortantes.

El ensayo toma una caña simplemente apoyada en sus extremos, aplicándole cargas puntuales aproximadamente en una distancia equivalente a la tercera parte de la longitud total de la muestra medida desde los extremos. Los apoyos de los extremos deben ser articulados para que permitan la rotación, y no exista ni desplazamiento vertical ni horizontal en esos puntos de apoyo. La carga $P$ del gato se aplica sobre una viga metálica, la cual descansa sobre los puntos de aplicación de las cargas. Es una prueba de flexión de 8 puntos.

Se colocaron tres deformímetros, uno en el centro para poder medir la deformación que sufre la caña Guadua a medida que se aumenta la carga aplicada. También se colocaron deformímetros a cada extremo para saber cuánto se asentaba la caña debido a que estaba asentada en cauchos sobre los soportes para tener mayor estabilidad. A la medida del deformímetro central se le resta el promedio de las medidas de los deformímetros de los extremos para poder determinar la medida exacta de la deformación que ocasiona la flexión por la carga aplicada en los 8 puntos.

Al ejecutar el ensayo de flexión de 8 puntos de carga puntual en una caña Guadua, el gato aplica una fuerza $P$ que se transmite a la viga metálica y se divide en fuerzas $\mathrm{P} / 4$ sobre los apoyos centrales de aplicación de las cargas. Se utilizaron los deformímetros para poder medir las deformaciones a medida que se aumenta la carga aplicada a la caña Guadua y poder medir su deformación máxima cuando se llega al máximo esfuerzo por flexión, tal como se observa en la figura 4. 


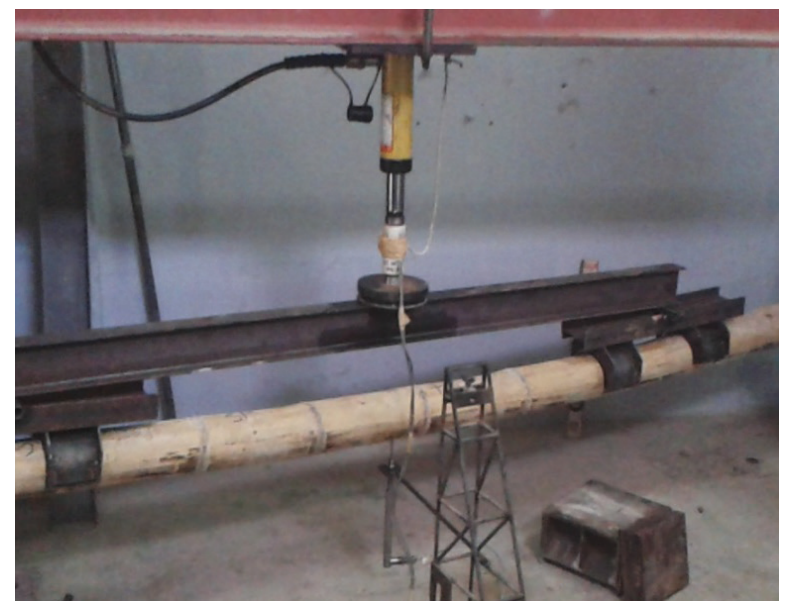

Figura 4. Caña Guadua pandeada antes de llegar a la falla debido a las cargas de flexión.

Fuente: Córdova, P. (2014). Obtención de las propiedades mecánicas y estructurales de la caña Guadua Angustifolia Kunth del Ecuador.

En el mecanismo de falla a flexión de la caña guadua, existe una rotura por compresión de las fibras superiores debido a que la lignina que une las fibras se rompe. Las fibras sometidas a tensión no fallan debido a que son más resistentes. La caña Guadua se pandea antes de llegar a la rotura debido a las cargas de flexión (figura 5).

Figura 5. Mecanismo de falla a flexión de la caña Guadua

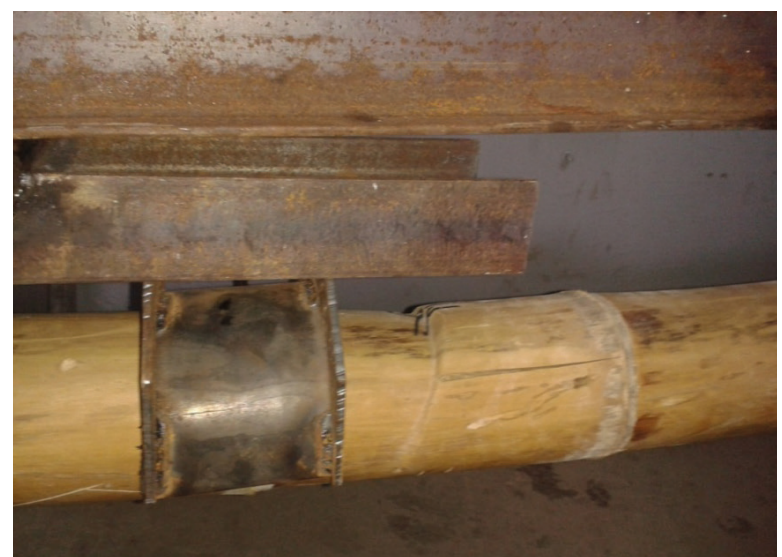

Fuente: Córdova, P. (2014). Obtención de las propiedades mecánicas y estructurales de la caña Guadua Angustifolia Kunth del Ecuador.

La figura 6 muestra en qué consiste un ensayo de flexión de 8 puntos de carga puntual en una caña Guadua. El gato aplica una fuerza P que se transmite a la viga metálica y se divide en fuerzas $\mathrm{P} / 4$ sobre los apoyos centrales de aplicación de las cargas. Se utilizaron los deformímetros para poder medir las deformaciones a medida que se aumenta la carga aplicada a la caña Guadua y poder medir su deformación máxima cuando se llega al máximo esfuerzo por flexión.

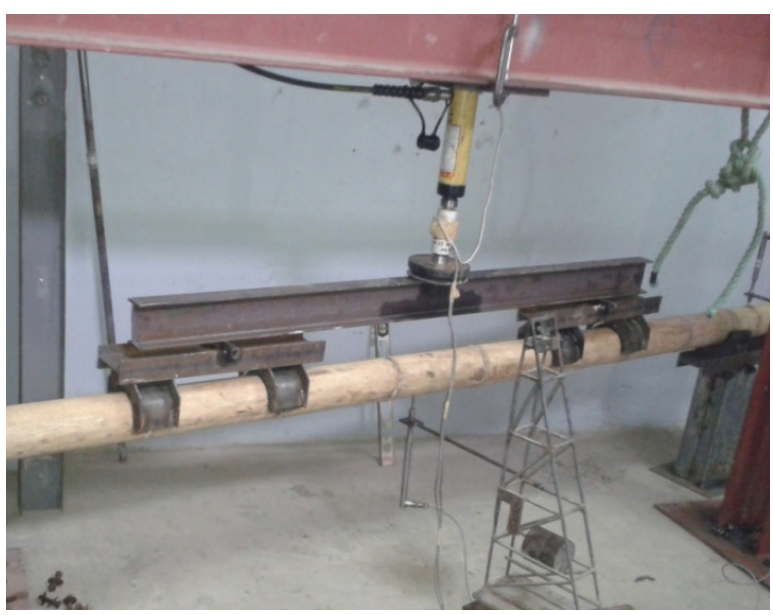

Figura 6. Ensayo de flexión de 8 puntos.

Fuente: Córdova, P. (2014). Obtención de las propiedades mecánicas y estructurales de la caña Guadua Angustifolia Kunth del Ecuador.

RESULTADOS DE LAS PRUEBAS DE CORTE La tabla 1 muestra las propiedades geométricas de las muestras de ensayos de resistencia al esfuerzo cortante y los resultados obtenidos de las pruebas. En la tabla 2 se puede apreciar el análisis estadístico de los resultados. Por su parte, la figura 7 presenta la variabilidad de los resultados.

\begin{tabular}{|c|c|c|c|c|c|c|c|c|}
\hline Muestra N & $\begin{array}{l}\text { Dext } \\
\text { (cm) }\end{array}$ & $\begin{array}{l}\text { D int } \\
\text { (cm) }\end{array}$ & $\begin{array}{l}\text { Esp } \\
\text { (cm) }\end{array}$ & $\begin{array}{l}\text { Altura de } \\
\text { muestra } \\
\text { (cm) }\end{array}$ & $\begin{array}{r}\text { Peso } \\
\text { (g) }\end{array}$ & $\begin{array}{r}\text { Area de } \\
\text { corte } \\
\text { (cm2) }\end{array}$ & $\begin{array}{r}P \\
\text { (KN) }\end{array}$ & $\begin{array}{r}\text { t ult } \\
\text { (Mpa) }\end{array}$ \\
\hline 1 (nudo) & 11,55 & 8,21 & 1,62 & 11,55 & 664,5 & 74,844 & 65,4 & 8,738 \\
\hline 2 (nudo) & 11,39 & 7,85 & 1,62 & 11,39 & 667,9 & 73,807 & 59,5 & 8,062 \\
\hline 3 (nudo) & 11,65 & 8,71 & 1,31 & 11,65 & 512,3 & 61,046 & 54,4 & 8,911 \\
\hline 4 (nudo) & 9,76 & 6,3 & 1,47 & 9,76 & 303,1 & 57,3888 & 60,6 & 10,560 \\
\hline 5 (nudo) & 10,16 & 8,45 & 0,82 & 10,16 & 251,7 & 33,3248 & 35,6 & 10,683 \\
\hline 6 (nudo) & 10,64 & 9,03 & 0,81 & 10,64 & 273,4 & 34,4736 & 40,8 & 11,835 \\
\hline 7 (nudo) & 9,97 & 8,18 & 0,97 & 9,97 & 245,4 & 38,6836 & 35,6 & 9,203 \\
\hline 8 & 10,24 & 8,54 & 0,84 & 10,24 & 198,9 & 34,4064 & 33,4 & 9,707 \\
\hline 9 & 11,42 & 9,29 & 1,07 & 11,42 & 324,3 & 48,8776 & 41,7 & 8,532 \\
\hline 10 (nudo) & 9,86 & 8,15 & 0,74 & 9,86 & 175,5 & 29,1856 & 25,5 & 8,737 \\
\hline 11 (nudo) & 9,67 & 6,5 & 1,41 & 9,67 & 274,9 & 54,5388 & 42,65 & 7,820 \\
\hline 12 (nudo) & 10,04 & 8 & 1,11 & 10,04 & 271,8 & 44,5776 & 49,4 & 11,387 \\
\hline 13 & 9,71 & 6,37 & 1,42 & 9,71 & 272,2 & 55,1528 & 62,8 & 11,387 \\
\hline 14 & 10,22 & 8,63 & 0,74 & 10,22 & 180,1 & 30,2512 & 29 & 9,586 \\
\hline 15 & 9,9 & 8,14 & 0,88 & 9,9 & 221,1 & 34,848 & 32,98 & 9,464 \\
\hline
\end{tabular}

Fuente: Córdova, P. (2014). Obtención de las propiedades mecánicas y estructurales de la caña Guadua Angustifolia Kunth del Ecuador. 
tABLA 2. ANÁLISIS ESTADÍSTICO DEL ESFUERZO ÚltIMO DE FUERZAS CORTANTES

\begin{tabular}{|c|c|}
\hline I ult Media= & $9,620 \mathrm{Mpa}$ \\
\hline Desviación estantadar $(\mathrm{S})=$ & 1,233 Мра \\
\hline$\tau k=$ & 7,172 Мра \\
\hline S/ $/$ ult Media= & 0,128 \\
\hline
\end{tabular}

Fuente: Córdova, P. (2014). Obtención de las propiedades mecánicas y estructurales de la caña Guadua Angustifolia Kunth del Ecuador.

Histograma y Polígono de frecuencias

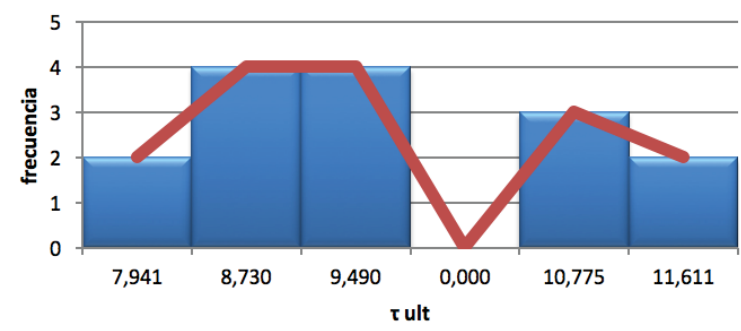

Figura 7. Análisis estadístico del esfuerzo cortante último de la caña Guadua. Fuente: Córdova, P. (2014). Obtención de las propiedades mecánicas y estructurales de la caña Guadua Angustifolia Kunth del Ecuador.

RESULTADOS DE LAS PRUEBAS DE FLEXIÓN TABLA 3. PROPIEDADES GEOMÉTRICAS DE LAS MUESTRAS DE CAÑA GUADUA PARA EL ENSAYO DE FLEXIÓN

\begin{tabular}{rrrrrrr}
\hline MUESTRA N & $\begin{array}{r}\text { D EXT 1 } \\
\text { (CM) }\end{array}$ & $\begin{array}{r}\text { D INT 1 } \\
\text { (CM) }\end{array}$ & $\begin{array}{r}\text { ESP 1 } \\
\text { (CM) }\end{array}$ & $\begin{array}{r}\text { DM 1 } \\
\text { (CM) }\end{array}$ & $\begin{array}{r}\text { ESPI } \\
\text { DEXT 1 }\end{array}$ & $\begin{array}{r}\text { I1 } \\
\text { (CM4) }\end{array}$ \\
\hline 1 & 11.83 & 8,22 & 1,81 & 10,025 & 0,1530 & 737,3 \\
\hline 2 & 10,73 & 8,95 & 0,94 & 9,84 & 0.0876 & 335,72 \\
\hline 3 & 11,9 & 9,11 & 1,62 & 10,505 & 0,1361 & 646,27 \\
\hline 4 & 10,06 & 8 & 0,81 & 9,03 & 0,0805 & 301,7 \\
\hline 5 & 11.57 & 9,09 & 1,17 & 10,33 & 0,1011 & 544,5 \\
\hline 6 & 11,3 & 9,1 & 1,15 & 10,2 & 0,1018 & 463,74 \\
\hline 7 & 10,98 & 8,37 & 1,44 & 9,675 & 0,1311 & 472,56 \\
\hline 8 & 11,19 & 8,09 & 1,38 & 9,64 & 0,1233 & 559,38 \\
\hline 9 & 11,32 & 8,19 & 1,73 & 9,755 & 0,1528 & 585,18 \\
\hline 10 & 10,04 & 8,46 & 0,83 & 9,25 & 0,0827 & 247,33 \\
\hline
\end{tabular}

Fuente: Córdova, P. (2014). Obtención de las propiedades mecánicas y estructurales de la caña Guadua Angustifolia Kunth del Ecuador.

tABLA 4. PROPIEDADES GeOMÉtricAS DE LAS MUESTRAS DE CAÑA GUADUA PARA EL ENSAYO DE FLEXIÓN

\begin{tabular}{rrrrrrr} 
MUESTRA N & $\begin{array}{r}\text { D EXT 2 } \\
\text { (CM) }\end{array}$ & $\begin{array}{r}\text { D INT 2 } \\
\text { (CM) }\end{array}$ & $\begin{array}{r}\text { ESP 2 } \\
\text { (CM) }\end{array}$ & $\begin{array}{r}\text { DM 2 } \\
\text { (CM) }\end{array}$ & $\begin{array}{r}\text { ESP/ } \\
\text { DEXT2 }\end{array}$ & $\begin{array}{r}\text { I 2 } \\
\text { (CML) }\end{array}$ \\
\hline 1 & 10,9 & 8,68 & 1,11 & 9,79 & 0,1018 & 414,27 \\
2 & 11,7 & 8,88 & 1,54 & 10,29 & 0,1316 & 614,62 \\
3 & 11,78 & 9,42 & 1,09 & 10,595 & 0,0925 & 560,38 \\
4 & 10,84 & 9,23 & 0,84 & 10,035 & 0,0775 & 321,51 \\
\hline 5 & 11,62 & 9,64 & 1,03 & 10,63 & 0,0886 & 471,03 \\
\hline 6 & 11,48 & 7,3 & 2 & 9,39 & 0,1742 & 713,18 \\
\hline 7 & 11,18 & 9,4 & 0,9 & 10,29 & 0,0805 & 388,65 \\
8 & 10,28 & 8,45 & 1,14 & 9,365 & 0,1109 & 297,94 \\
9 & 9,74 & 7,84 & 0,96 & 8,79 & 0,0986 & 256,33 \\
10 & 11,68 & 9,09 & 1,3 & 10,385 & 0,1113 & 578,43 \\
\hline
\end{tabular}

Fuente: Córdova, P. (2014). Obtención de las propiedades mecánicas y estructurales de la caña Guadua Angustifolia Kunth del Ecuador.
tABLA 5. PROPIEDADES geOMÉTRICAS DE LAS MUESTRAS DE CAÑA GUADUA PARA EL ENSAYO DE FLEXIÓN

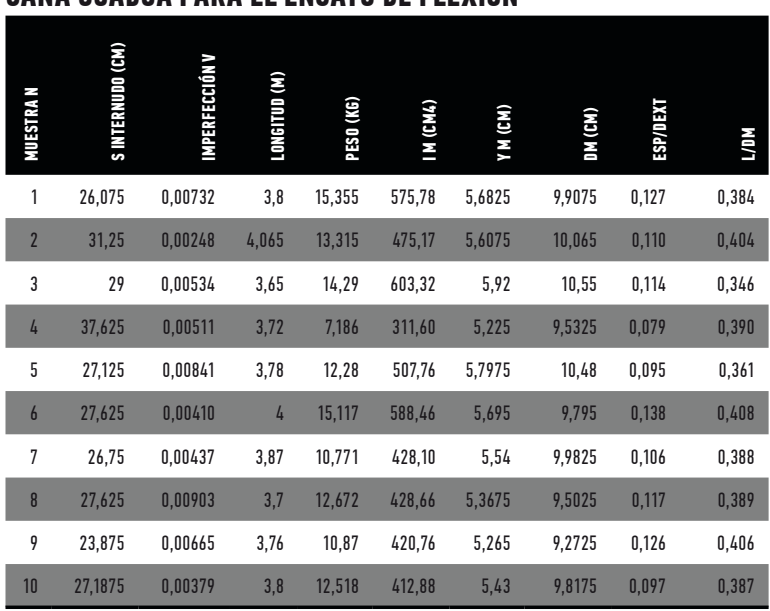

Fuente: Córdova, P. (2014). Obtención de las propiedades mecánicas y estructurales de la caña Guadua Angustifolia Kunth del Ecuador.

Se midió cada una de las propiedades en ambos extremos de la muestra de caña Guadua que se iba someter al ensayo de flexión. En las muestras se pudo determinar la variación que existe en estas propiedades de un extremo a otro.

Las tablas 3, 4 y 5 muestran las mediciones de las propiedades geométricas de las cañas a ensayarse. La tabla 6 muestra las longitudes de los diferentes tramos de la muestra representada en la figura 8.

TABLA 6. LONGITUDES DE LAS LUCES LIBRES DE LAS MUESTRAS DE CAÑA GUADUA PARA EL ENSAYO DE FLEXIÓN

\begin{tabular}{|rrrrrrrrrr}
\hline & & & & & & & & & \\
\hline 1 & 0,97 & 1,39 & 0,98 & 0,21 & 0,28 & 0,23 & 0,26 & 0,32 & 0,34 \\
\hline 2 & 1,21 & 1,36 & 0,89 & 0,42 & 0,22 & 0,39 & 0,32 & 0,38 & 0,27 \\
\hline 3 & 0,8 & 1,45 & 1 & 0,23 & 0,22 & 0,33 & 0,31 & 0,27 & 0,25 \\
\hline 4 & 1,14 & 1,11 & 0,76 & 0,4 & 0,32 & 0,39 & 0,38 & 0,37 & 0,37 \\
\hline 5 & 0,69 & 1,72 & 0,97 & 0,16 & 0,27 & 0,2 & 0,26 & 0,32 & 0,32 \\
\hline 6 & 1,25 & 1,32 & 0,99 & 0,25 & 0,19 & 0,23 & 0,25 & 0,24 & 0,33 \\
\hline 7 & 0,86 & 1,6 & 1,03 & 0,15 & 0,22 & 0,2 & 0,25 & 0,33 & 0,32 \\
\hline 8 & 0,76 & 1,63 & 0,93 & 0,21 & 0,26 & 0,24 & 0,25 & 0,31 & 0,34 \\
\hline 9 & 0,93 & 1,19 & 1,11 & 0,2 & 0,28 & 0,18 & 0,23 & 0,25 & 0,3 \\
\hline 10 & 1,05 & 1,16 & 0,21 & 0,21 & 0,25 & 0,25 & 0,28 & 0,29 & 0,3 \\
\hline
\end{tabular}

Fuente: Córdova, P. (2014). Obtención de las propiedades mecánicas y estructurales de la caña Guadua Angustifolia Kunth del Ecuador. 


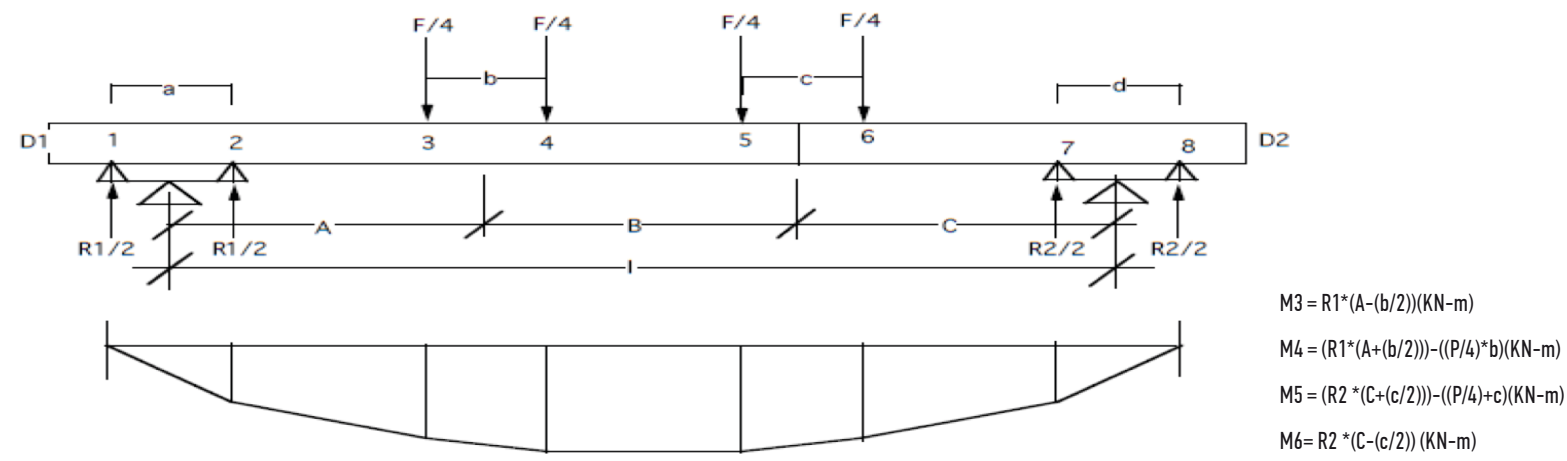

Figura 8. Esquema del ensayo de flexión en una muestra de caña Guadua. Gráfico del momento flector

Fuente: Córdova, P. (2014). Obtención de las propiedades mecánicas y estructurales de la caña Guadua Angustifolia Kunth del Ecuador.

TABLA 7. RESULTADOS DE LOS ENSAYOS Y CÁLCULO DEL ESFUERZO DE FLEXIÓN ÚLTIMA DE CAÑA GUADUA

\begin{tabular}{rrrrrrrrrrr}
\hline MUESTRA N & \multicolumn{1}{c}{$\mathbf{P}$ (KN) } & R1 (KN) & R2 (KN-M) & M3 (KN-M) & M4 (KN-M) & M5 (KN-M) & M6 (KN-M) & M MAX (KN-M) & O MAX (MPA) \\
\hline 1 & 13,95 & 6,996 & 6,954 & 5,877 & 6,789 & 6,812 & 5,702 & 6,812 & 67,226 \\
\hline 2 & 12,35 & 5,604 & 6,746 & 5,884 & 6,689 & 6,113 & 4,722 & 6,689 & 78,942 \\
\hline 3 & 16,55 & 8,784 & 7,766 & 5,666 & 7,106 & 7,697 & 6,717 & 7,697 & 75,526 \\
\hline 4 & 6,25 & 2,730 & 3,520 & 2,594 & 3,038 & 2,748 & 2,024 & 3,038 & 50,938 \\
\hline 5 & 12,35 & 6,687 & 5,663 & 3,744 & 4,680 & 5,412 & 4,587 & 5,412 & 61,79 \\
\hline 6 & 11,5 & 5,330 & 6,170 & 5,996 & 6,610 & 6,159 & 5,368 & 6,610 & 63,971 \\
\hline 7 & 10,9 & 5,715 & 5,185 & 4,201 & 4,948 & 5,296 & 4,485 & 5,296 & 68,538 \\
\hline 8 & 13,8 & 7,253 & 6,547 & 4,606 & 5,557 & 6,034 & 5,074 & 6,034 & 75,551 \\
\hline 9 & 8,7 & 4,592 & 4,108 & 3,743 & 4,299 & 4,529 & 4,046 & 4,529 & 56,674 \\
10 & 7,55 & 3,920 & 3,630 & 3,567 & 4,136 & 4,263 & 3,757 & 4,263 & 56,061 \\
\hline
\end{tabular}

Fuente: Córdova, P. (2014). Obtención de las propiedades mecánicas y estructurales de la caña Guadua Angustifolia Kunth del Ecuador.

Figura 9. Análisis estadístico del esfuerzo a flexión último de la caña Guadua.

\section{Histograma y Polígono de frecuencias}

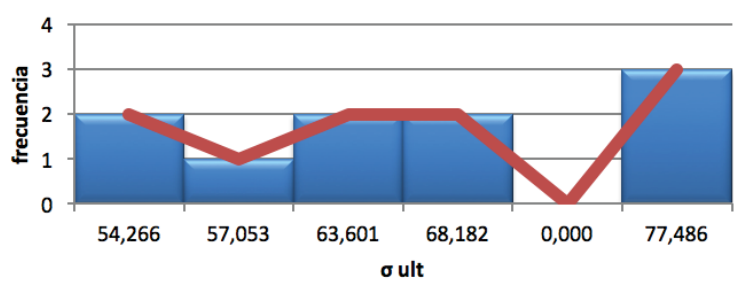

Fuente: Córdova, P. (2014). Obtención de las propiedades mecánicas y estructurales de la caña Guadua Angustifolia Kunth del Ecuador.

Se obtuvo una resistencia característica a la flexión última de $45.85 \mathrm{MPa}$, lo que es igual a $467.38 \mathrm{~kg} / \mathrm{cm} 2$, y soportaron en promedio un momento flector de 55.3 Ton-m y una deflexión máxima promedio en el centro de $9.5 \mathrm{~cm}$, tal como lo muestran los resultados de las tablas 7 y 8.
Tiene una gran resistencia a la flexión pero se deforma mucho lo que impide cumplir con condiciones mínimas de servicio $(\mathrm{L} / 400=4 / 200$ $=0.02 \%$ ) cuando está sometida a su máxima resistencia a la flexión. Cuando la caña está sometida a flexión y llega a $2 \mathrm{~cm}$ de deformación central, en promedio resiste un esfuerzo flector de $18.86 \mathrm{MPa}$, lo cual es aproximadamente 2.7 veces menos su máxima capacidad. Esto puede ayudarnos en la búsqueda de un factor de seguridad para el esfuerzo admisible.

La figura 9 ilustra la variabilidad de los resultados a través de la distribución de frecuencias. Las curvas esfuerzo-deformación fueron elaboradas tal como se muestra en la Figura 10. Estas permitieron el cálculo del módulo de elasticidad como se muestra en la tabla 9 y el análisis estadístico como se aprecia en la tabla 10. 
TABLA 9. CÁLCULO DEL MÓdULO DE ELASTICIDAD CUANDO SE APLICA EL 50\% DEL ESFUERZO ÚLTIMO A FLEXIÓN

\begin{tabular}{|c|c|c|c|c|c|c|c|c|c|}
\hline MUESTRA N & $0.5 \mathrm{P}(\mathrm{I}$ & & R1(N) & R2ULT (N) & $\begin{array}{l}(A / 2)+A+(B / 2) \\
(M)\end{array}$ & $I(M L)$ & $\begin{array}{l}\text { S(0.5P) CENTRO } \\
(\mathrm{M})\end{array}$ & M CENTRO (N-M) & $\begin{array}{l}\mathrm{E}(0.5) \\
\text { (GPA) }\end{array}$ \\
\hline 1 & 1 & 7000 & 3510,48 & 3489,52 & 1,78 & 0,00000576 & 0,0381 & 3816,15 & 38,37 \\
\hline 2 & & 6350 & 2881,36 & 3468,64 & 2,09 & 0,00000475 & 0,0412 & 3848,63 & 65,36 \\
\hline 3 & & 8275 & 2757,59 & 2757,59 & 1,69 & 0,00000503 & & 1660,64 & \\
\hline 4 & & 3250 & 1419,95 & 1830,15 & 1,89 & 0,00000312 & 0,0296 & 1781,64 & 59,53 \\
\hline 5 & & 6300 & 3410,95 & 2889,05 & 1,65 & 0,00000508 & 0,0336 & 2919,06 & 29,42 \\
\hline 6 & & 5850 & 2711,17 & 3138,62 & 2,03 & 0,00000588 & 0,0338 & 3560,04 & 53,44 \\
\hline 7 & & 5550 & 2910,17 & 2649,62 & 1,76 & 0,00000428 & 0,0424 & 2901,9 & 32,17 \\
\hline 8 & B & 6850 & 3600,38 & 3249,62 & 1,70 & 0,00000429 & 0,0363 & 3311,26 & 40,18 \\
\hline 9 & 9 & 4450 & 2348,99 & 2101.01 & 1,62 & 0,00000421 & 0,0327 & 2468,75 & 31,01 \\
\hline 10 & & 3900 & 2024,78 & 1875,22 & 1,76 & 0,00000413 & 0,0313 & 2422,49 & 39,94 \\
\hline
\end{tabular}

Fuente: Córdova, P. (2014). Obtención de las propiedades mecánicas y estructurales de la caña Guadua Angustifolia Kunth del Ecuador.

$\sigma(\mathrm{MPa})-\delta(\mathrm{cm})(5-\mathrm{Flex})$

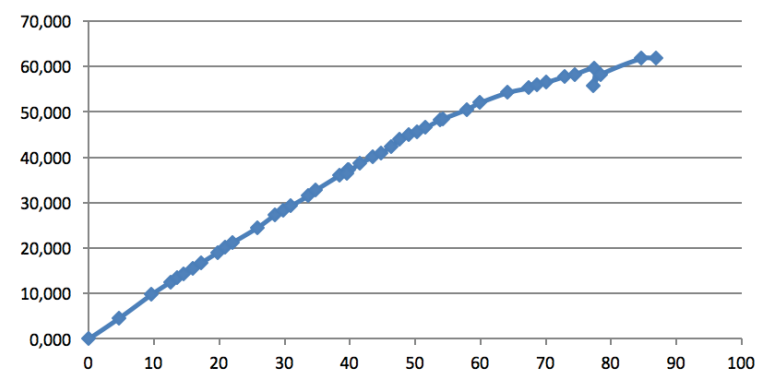

Figura 10. Curva del esfuerzo de flexión vs. la deformación en el centro de la muestra Flex5.

Fuente: Córdova, P. (2014). Obtención de las propiedades mecánicas y estructurales de la caña Guadua Angustifolia Kunth del Ecuador

TABLA 10. ANÁLISIS ESTADÍSTICO DEL MÓDULO DE ELASTICIDAD DEL 50\% DEL ESFUERZO ÚLTIMO DE CAÑA GUADUA A FLEXIÓN

\begin{tabular}{lll}
$\mathrm{E}(0.5 \mathrm{P})$ Media $=$ & 38.943 & Мpa \\
Desviación estantadar $(\mathrm{S})=$ & 13.066 & Мpa \\
$\mathrm{E}(0.5 \mathrm{P}) \mathrm{K}=$ & 12.157 & Мра \\
$\mathrm{S} / \mathrm{E}(0.5 \mathrm{p})$ Media= & 0.336 & \\
\hline
\end{tabular}

Fuente: Córdova, P. (2014). Obtención de las propiedades mecánicas y estructurales de la caña Guadua Angustifolia Kunth del Ecuador.

Este método está basado en el colorario de Mohr, a partir del método de la doble integración del momento flector en el punto de estudio de la viga determinar la deformación en ese punto; es decir $\delta(x)=\int \frac{M(x)}{E l}(d x)$. Para la configuración de nuestra viga simple con cuatro cargas iguales pero no ubicadas simétricamente sobre la viga, lo que resulta para nuestro estudio en el punto central de la viga: $E=\iint \frac{M(x)}{\delta I}(d x)$, la cual será la fórmula que utilizaremos para nuestro estudio del módulo de elasticidad de la caña Guadua.

Se calcula la deformación que tiene la viga en el centro, esto es $x=\frac{a}{2}+A+\frac{B}{2}$, cuando se aplica el $50 \%$ del esfuerzo último a flexión.
DISCUSIÓN DE RESULTADOS

En el análisis estadístico hecho a los resultados producto de los ensayos de resistencia al esfuerzo cortante de caña Guadua se concluye que esta es la propiedad mecánica más débil que tienen las caña Guadua. Además se obtuvieron resultados bastantes uniformes en esta propiedad.

De los resultados de las pruebas de la resistencia al corte, se determina que a medida que el espesor de la pared de la caña y el área sometida a corte son mayores, mayor va a ser la resistencia al esfuerzo cortante que va a tener la caña Guadua. Así mismo, se determina que a medida que la relación espesor/diámetro externo es mayor, la resistencia al esfuerzo cortante va a ser mayor.

En el análisis estadístico hecho a los resultados, producto de los ensayos de resistencia al esfuerzo de flexión de caña Guadua, se concluye que, cuando se aplican cargas muy altas perpendicularmente a una caña se la somete a altas deformaciones, debido a su alta flexibilidad lo que ocasiona que la estructura no pueda cumplir los requisitos de serviciabilidad cuando está sometida a su máxima resistencia a la flexión. Este hecho es una desventaja, pero una ventaja a la vez. Es decir, es frustrante el tener que diseñar estructuras de caña Guadua a flexión sometidas a cargas muy bajas en comparación a su capacidad de resistencia real, pero esto es positivo porque se cuenta con la seguridad que las cargas no harán fallar a la estructura.

De la comparación de los diferentes resultados entre las pruebas que se realizaron, como se esperaba, la falla de flexión está dominada por la resistencia a la compresión. Para otros materiales se tiene: $\mathrm{Rflex}=1.2 \mathrm{RComp}$, en el caso de los ensayos de este trabajo resultó: 45.85/37.76 = 
1.21. Por supuesto, estos resultados se basan en un bajo número de muestras.

Para obtener una resistencia mayor a la flexión en las caña Guadua se tiene que contar con un momento de inercia bajo y un radio de giro alto, esto se puede lograr teniendo una relación entre el espesor y el diámetro exterior (Esp/Dext) alta, puesto que la inercia depende directamente del espesor y del diámetro exterior, y el radio de giro es igual a la mitad del diámetro exterior.

Para futuras investigaciones se debe tomar en cuenta en el análisis estadístico de las propiedades geométricas de la caña Guadua, la influencia de la conicidad, es decir, la forma de cono truncado que tiene ésta por sus cambios de diámetro a lo largo de ésta.

En el estudio de la flexión en cañax Guadua, se puede hacer ensayos con cargas permanentes durante períodos largos, para determinar las deformaciones a largo plazo que sufre con cargas altas que no la lleven a su falla última instantáneamente. Deberían hacerse estudios de flexocompresión a muestras de caña Guadua largas para determinar su comportamiento y resistencia como columnas.

\section{REFERENCIAS BIBLIOGRÁFICAS}

Amada, S., Untao, S. 2001. "Fracture properties of bamboo". Part B 32 (2001) 451-459.

Asociación Colombiana de Ingeniería Sísmica, Normas Colombianas de diseño sismorresistente

Conference on Timber Engineering 2010 (NSR-98), Asociación colombiana de Ingeniería Sísmica, Bogotá, Colombia, 1997.

Córdova, P. (2014). Obtención de las propiedades mecánicas y estructurales de la caña Guadua Angustifolia Kunth del Ecuador. (Trabajo de Grado). Facultad de Ingeniería, Universidad Católica de Santiago de Guayaquil, Guayaquil.

David J. A. Trujillo "Axially Loaded Connections In Guadua Bamboo" Proceedings of the Nocmat 2009, Bath, UK.
E. Dutta et. Al "Utilization aspects of bamboo and its market value" Indian Forest March-2008.

Fotografie Studio Zuarq - Bogotà www.zuarq. com.

Ghavami, K. et al. 2003. "Bamboo: Functionally Graded Composite Material." Asian Journ. of Civil Eng.Vol. 4, No. 1 (2003).

Chavami, K. 2005. "Bamboo as reinforcement in structural concrete elements." Cement \& Concrete Composites 27 (2005).

J. Coreal et at "Structural Behavior of Glued Laminated Guadua Bamboo As A Construction Material" World Conference on Timber Engineering 2010.

L. Osorio, E.Trujillo, A.W. Van Vuure, F. Lens, J. Ivens, I. Verpoest "The Relation Between Bamboo Fibre Microstructure And Mechanical Properties" 14th European Conference On Composite Materials - Budapest, Hungary.

La Tegola, A., Mera, W. (2012). Diseño de las estructuras de hormigón armado a los estados límites. Universidad Católica de Santiago de Guayaquil.

T.M. Obermann, R. Laude "Bamboo Poles For Spatial And Light Structures" -Bamboo Space Research Project Universidad Nacional de Colombia 166_OB.

T. Tada, K. Hashimoto \& A. Shimabukuro "On characteristics of bamboo as structural materials" in Challenges, Opportunities and Solutions in Structural Engineering and Construction - Chafoori (ed.) 2010.

Wan Tarmeze Wan Ariffin "Numerical Analysis of Bamboo and Laminated Bamboo Strip Lumber" Research Project 2002-2005 - Sponsor Government of Malaysia.

Yao, W. \& Li, Z. 2003. "Flexural behavior of bamboo-fiberreinforced mortar laminates". Cement \& Concrete Research 33 (2003). 